\title{
Ranking Risk Exposures for Situational Surveillance of Falls with Sensors
}

\author{
Alla Kammerdiner
}

Received: June 29, 2015/ Accepted: date

\begin{abstract}
Falls pose a significant public health problem. The usefulness of wearable sensors for detection of falls is well-known. We propose a novel optimization-based approach that was formulated for ranking exposures to falls using information streamed from multiple sensors. Our method incorporates statistical estimation. We illustrate our technique on synthetic data. Future research is aimed at assessing the validity of this new method.
\end{abstract}

Keywords Combinatorial optimization · estimation · sensor-based surveillance $\cdot$ falls $\cdot$ risk assessment $\cdot$ personalized health

\section{Introduction}

In this paper we develop and describe a novel optimization method for sensorbased surveillance and monitoring of hazardous situations that may result in falling. In this approach, the focus is shifted from traditional falls detection to situational assessment related to falls. Falls can arise in variety of situations when a person looses balance and fails to regain it. For instance, either trips or slips may lead to a loss of balance that could possibly result in a fall. The ability to distinguish various kinds of exposures to falls could potentially be used for acquiring the personalized data on situational risk of falls. Furthermore, the capacity for differentiating among exposures to falls can be utilized in new technologies for mobility assistance and falls prevention. Accordingly, the longterm objective of this research is to enable smart sensor-based technologies for recognizing and differentiating among various types of exposures to falls.

A. Kammerdiner

New Mexico State University, PO Box 30001, MSC 4230, Las Cruces, NM 88001

Tel.: +157-56-462475

Fax: +157-56-462479

E-mail: alla@nmsu.edu

(C) 2015. This manuscript version is made available under the Elsevier user license http://www.elsevier.com/open-access/userlicense/1.0/ 
The presented new approach for ranking situational exposures to falls is general, and not specific to age-related falls. However, falls in older adults serve as a long-term motivation behind this research, since older population is disproportionably affected by falls. Falls in elderly is a globally recognized public health problem that is projected to worsen due to the worldwide population aging [1]. In the United States, a third of adults 65 years and older falls each year [2]. Falls in older adults result in reduced mobility, loss of functional independence, depression, trauma, and death. In healthy, active older adults situational and environmental factors may be predominant sources of falls risk [3].

A fall is commonly defined as an unintentional event that result in a person coming to rest on a ground, floor, or lower level and that excludes a seizure, heart attack, stroke or a major displacing force (e.g., an earthquake) [4]. A fall is said to happen in the following three phases [3]. The first phase is the initiating event or a perturbation that creates a loss of balance, i.e., displaces the center of mass beyond its base of support. The second phase is a failure to detect and correct this displacement in time to regain balance and avoid a fall. The third phase is the contact with the resting surface (e.g., ground or floor) causing the transmission of forces to organs and body tissue.

A near fall is traditionally defined as a loss of balance or stumble event that would result in a fall if sufficient recovery mechanisms were not activated [5]. This definition has been recently extended in [5] to include the activation of at least two of the fall-specific compensatory mechanisms. These may involve unplanned movement of arms or/and legs, unplanned change in stride length, lowering of the center of mass, unplanned change in stride velocity, and trunk tilt. The new definition of near falls helps improve differentiation of these events from obstacle negotiation [5]. Near falls are related to fall risk, occur more frequently than falls and may precede falls [6]. The frequency of near falls may provide a broader, more robust estimate of falls risk [5].

The first phase is common to falls and near falls. This phase begins with a perturbation or initiating event that involves external factors such as environmental hazards as well as physical activities in progress at the time of the event. For instance, different environmental hazards cause trips versus those that cause slips. Trips and slips are two commonly distinguished types of exposures to falls or near falls. While the absence of falls risk exposure include both the usual physical activities (such as walking) and the activities with obstacle avoidance. The ability to rank these in terms of their likelihood can be useful for gaining insights into situational and environmental risk factors of falls. Furthermore, a different fall prevention mechanism would be more suitable for trips versus slips. When combined with fall detection, the ranking of exposures could potentially enable more effective prevention.

Falls detection received and continues to attract a lot of attention from both researchers and practitioners $[7,8]$. The usefulness of wearable sensors for detection of falls is well-known. As reviewed in [8], many studies confirm usefulness of wearable sensors and sensor systems for detection of falls with the analytical methods ranging from simple thresholds [9] to advanced neural 
networks [10]. In practice, simple sensors are often used to monitor the activity of daily living (or ADL) and generate the alarms if no activity is detected at certain periods of time. Recent research indicates that an improved detection of falls is possible using the complementary information from sensors placed at different locations on a body [11].

The main contribution of this paper is a new analytical approach for sensorbased surveillance and risk assessment of falls. Most research work to-date deals with either sensor-based falls detection [7-9,12-16] or sensor-based assessment of overall falls risk [17-20]. In contrast, here we present a new mathematical framework for dealing with the situational assessment of falls risk exposures using multiple sensors. Wearable sensors (specifically, accelerometers) allow to record the information about motion of a human body that may be useful for understanding loss of balance $[8,21]$. Multiple sensors can be positioned on various locations of a body to potentially provide complimentary information [11]. The presented approach combines mathematical techniques to analyze the signals from motion sensors and rank distinct types of falls exposures.

We propose a distinctly new modeling framework for sensor-based monitoring of falls. Our framework combines statistical estimation with combinatorial optimization. We show how our approach can be applied on the simple example with two basic exposures and simplified synthetic data for two sensors. The presented example is not meant to demonstrate the validity of our approach. Additional research is needed to validate the proposed analytical approach on real data. Currently, we are collecting the data with a system of wearable sensors in human subject experiments. The acquired dataset will be used in future studies to assess the validity of the new optimization-based method.

The paper is organized as follows. In Section 2 we describe the proposed novel methodology for monitoring and risk assessment of falls. This section is split into two parts because the approach combines combinatorial optimization and statistical estimation to rank risk exposures from streams of sensor data. First, we formulate the task of ranking distinct types of risk exposures to falls as the problem of maximizing the likelihood of observed data. Second, we derive the formulas for the coefficients of the maximization problem and reduce the task of finding the coefficients to statistical estimation of certain density functions. The formulation of the maximization problem is presented in Section 2.1, followed by the methods for estimating the coefficients that are described in Section 2.2. In Section 3, a detailed example is provided to show how to use the approach. The example is not intended to demonstrate the validity. The validity will be addressed in future studies. For transparency and simplicity, we use artificial data as a very rough approximation of two sensor signals. The synthetic data are defined analytically, split into the past data (for training) and the future data (for testing), and used to rank likelihood of falls versus no-falls for future observations. Some clarifications on the new approach and the example, along with the limitations of this study and the future research directions are discussed in Section 4. Finally, in Section 5 we 
present the conclusions and discuss the advantage of our approach compared to other methods.

\section{Optimization and estimation-based methodology for ranking risks of falls from multiple sensors}

In this section we describe a new methodology for ranking distinct types of potential exposures to falls and near falls based on the past data. The methodology uses a combination of optimization and statistical approaches. Together, the approaches allow tracking these risk rankings in time and can potentially lead to the development of new devices for falls prevention.

\subsection{Formulation of the optimization problem}

Information technology plays increasing role in personalized health monitoring. Simple wearable devices equipped with sensors such as pedometers and activity trackers are used to monitor various characteristics, such as a number of steps taken, calorie expenditure, distance travelled, etc. These devices allow their users monitor their physical activity, and improve health and fitness. Exposure of adults to risks of falling can be monitored with sensor-based wearable devices.

In monitoring exposure to falls, a key surveillance challenge is to estimate the types of fall risk exposure (e.g., slip, trip, absence of exposure, etc.) present in the past, current, or future state of a person based on a sequence of data from one or more sensors. The focus of the present work is on the basic types of exposures, which can be identified with the sensors that generally provide kinematic information such as acceleration and balance.

Suppose that one or more sensors, either wireless or wired, are placed at various locations on the subject's body. This sensor or system of sensors produces a stream of data (or measurements), each with distinct time label. These data can be arranged into sequences of observations called time series, each corresponding to the specific sensor and ordered according to time labels. Formally, let $t_{k} \geq 0, k=1,2, \ldots$ be a sequence of times. Let $S$ denote the total number of sensors used, and let the index $s, s=1,2, \ldots, S$ represent the sensor number. At time $t_{k}$ each sensor $s$ has an $N$-dimensional noisy measurement vector $\left(x_{1}^{s}\left(t_{k}\right), x_{2}^{s}\left(t_{k}\right), \ldots, x_{N}^{s}\left(t_{k}\right)\right)=: \overline{X^{s}}\left(t_{k}\right)$ associated with it. Let $D$ denote the dimensionality of a combined vector of measurements from all $S$ sensors, then $D=N \times S$. In general, $D=\sum_{s=1}^{S} N_{s}$, where $N_{s}$ is the dimensionality of $s$-th sensor (e.g., a 3-D accelerometer has dimensionality 3 ).

In the human subject, we define the exposure to the risk of falling as the quantified potential for a sudden loss of balance that might occur as a result of some activity or event. We are interested in assessing the exposure to the risk of falling in the current state, where the exposure is determined or quantified based on potential for a sudden loss of balance to happen within some specified 
period of time in the future. We assume that this period starts from the current moment of time and call it the prediction period.

Suppose the prediction period is represented by time $\tau$.

Exposure to falls risk can be categorized in a variety of way depending on the goals of risk monitoring. For instance, preventive monitoring of falls risk situations may lead to the exposure to risks of fall be categorized based on the initiation of the fall exposure (e.g., right leg-initiated potential loss of balance or balance loss originating with left leg, etc.), or alternatively on the type of balance loss (e.g., potential slip vs potential trip, etc.). For a sake of brevity we will use risk exposure and risk interchangeably.

Let us consider $R$ types of risks (i.e., exposures to risks of falling), including the absence of exposure, which we will call a false alarm. Let $t$ be the current time. The goal is to (correctly) rank these $R$ risks (or risk exposures and false alarm(s)) in an decreasing order of their likelihood within the prediction time period $[t, t+\tau]$ based on the sensor(s) data.

Let $r, r=1,2, \ldots, R$, denote the type of exposure to risk of falling. Suppose that $r=1$ is a false alarm. For a fixed sensor $s$, let $\pi_{s}$ denote a permutation of $R$-element set that represent the ranking of risk exposures. Then

$$
\pi_{s}=\left(\begin{array}{c}
\pi_{s}(1) \\
\pi_{s}(2) \\
\vdots \\
\pi_{s}(R)
\end{array}\right)
$$

where $\pi_{s}(r)$ is the ranking of the risk $r$ according to the sensor measurements produced by sensor $s$.

Combining together all $S$ sensors, the association between the observations from $S$ sensors and their corresponding risk rankings can be represented as a $R \times S$ matrix with permutations of size $R$ as its columns:

$$
\Pi=\left(\begin{array}{cccc}
\pi_{1}(1) & \pi_{2}(1) & \ldots & \pi_{S}(1) \\
\pi_{1}(2) & \pi_{2}(2) & \ldots & \pi_{S}(2) \\
\vdots & \vdots & \ddots & \vdots \\
\pi_{1}(R) & \pi_{2}(R) & \ldots & \pi_{S}(R)
\end{array}\right)
$$

The data association problem for ranking risk exposures is the problem of determining the most likely association of the observations from sensors to the rankings of risk exposures and false alarm(s). Therefore, the task of ranking risk exposures can be posed as the following combinatorial optimization problem.

$$
\max _{\pi_{1}, \ldots, \pi_{S} \in \Pi_{R}} \sum_{r=1}^{R} L_{r \pi_{1}(r) \ldots \pi_{S}(r)},
$$

where $\Pi_{R}$ is the set of all permutations of $\{1,2, \ldots, R\}$, and the cost coefficients $L_{r i_{1} \ldots i_{S}}$ are the log-likelihood of risk $r$ having rankings $i_{s}$ for sensor $s$, $1 \leq s \leq S$. 
Remark 1. The problem formulation (2) requires that the distinct types of risk exposures and false alarm(s) are mutually independent. In particular, this is the case when the types of risks are based on mutually disjoint events. For instance, a trip excludes a slip, or a balance loss as a result of left leg being tripped excludes a loss of balance due to right leg tripped. Importantly, this assumption of independence is necessary in order to get a product of likelihoods leading to the sum of log-likelihoods shown in (2).

Remark 2. Formulation (2) is the multidimensional assignment problem [22, 23].

2.2 Estimation of the problem's coefficients from sensor data

For every given moment of time $t$, let us combine all the $N$-dimensional noisy measurement vectors $\overline{X^{s}}(t), s=1, \ldots, S$ into a common $(N \times S)$ dimensional data vector $\bar{X}(t):=\left(\overline{X^{1}}(t), \ldots, \overline{X^{S}}(t)\right)$. From a statistical standpoint, $\{\bar{X}(t), t \geq 0\}$ is a realization of some stochastic process $\{X(t), t \geq 0\}$ with an unknown probability distribution. If $\Pi$ is the true ranking of the types of risk exposures $r=1, \ldots, R$ on some time interval $\left(t_{0}, t_{1}\right]$. Then the distribution of this process $X(t)$ on that specific interval is defined by the unknown true ranking $\Pi$. Therefore, $\Pi$ is seen as the collection of distributional parameters of $X(t)$.

Here we use the maximum likelihood method [24,25] to estimate the values of $\Pi$. The maximum likelihood estimation, or MLE, is the statistical procedure for finding the parameters of an unknown distribution so that the likelihood function is maximized. Loosely speaking, the likelihood of a data sample is the probability of obtaining that particular data, given the chosen probability distribution model $[26]$. Let $f\left(\bar{X}(t) \mid i_{1}, \ldots, i_{S} ; r\right)$ denote the probability density function $(\mathrm{PDF})$ that specifies the probability of observing data $\bar{X}(t)$ given that the parameters in the $r$-th row of $\Pi$ are $i_{1}, \ldots, i_{S}$, ordered as listed here. Then the coefficients $L$ in (2) can be estimated from data as follows:

$$
L_{r i_{1} \ldots i_{S}}=\ln f\left(\bar{X}(t) \mid i_{1}, \ldots, i_{S} ; r\right)=\ln f\left(\bar{X}(t) \mid \pi_{s}(r)=i_{s}, 1 \leq s \leq S\right) .
$$

Let us define trigger time $T$ as the moment of time when an event happens that triggers a loss of balance, which may or may not result in a fall. Furthermore, let fallout time $H$ denote the moment when a loss of balance ends (either in a fall or regaining of balance). Note that $H>T$, since each trigger time precedes the respective fallout time $H$. We find the coefficients $L$ in (3) by analyzing sensor data $\bar{X}(t)$ with $t \in(T-\tau, H)$ and the respective outcomes at time $H$. Specifically, for different types of risk exposure $r=1, \ldots, R$ and fixed ranking assignments $i_{1}, \ldots, i_{S}$, the coefficients $L$ can be determined using non-parametric estimators of density functions $f\left(\bar{X}(t) \mid i_{1}, \ldots, i_{S} ; r\right)$.

Two simple estimators are the histogram and the rectangle kernel. Both produce rather non-smooth density functions. 
Let $E=[0, P]$ denote the estimation interval (or training interval). The histogram estimator of $f\left(X(t) \mid i_{1}, \ldots, i_{S} ; r\right)$ is defined as

$$
\widehat{f}\left(X(t) \mid i_{1}, \ldots, i_{S} ; r\right)=\frac{1}{P} \sum_{k=1}^{K} \frac{N_{k}}{\mu\left(A_{k}\right)} \mathbf{I}_{A_{k}}(X(t)),
$$

where the support of $f\left(\bar{X}(t) \mid i_{1}, \ldots, i_{S} ; r\right)$ is partitioned into a sequence of hyperrectangles $A_{1}, \ldots, A_{K}$ such that each $A_{k} \subset \mathbf{R}^{(N \times S)}$ for $k=1, \ldots, K$ and their disjoint union is the $\operatorname{support} \operatorname{supp}(f)=\uplus_{k=1}^{K} A_{k}$. In (4), $N_{k}$ denotes the number of observations in the hyperrectangle $A_{k}, \mu(\cdot)$ is a Lebesgue measure in $\mathbf{R}^{(N \times S)}$ (i.e., the $(N \times S)$-dimensional volume of $\left.A_{k}\right)$, and $\mathbf{I}_{A_{k}}(\cdot)$ is an indicator function on $A_{k}$. Practical algorithms for constructing multidimensional histograms from continuous data streams are described in [27].

Let $x \in \mathbf{R}^{(N \times S)}$. The rectangular kernel estimator of $f\left(X(t) \mid i_{1}, \ldots, i_{S} ; r\right)$ is defined below by introducing the hyperrectangular windows $A$ with volume $h^{(N \times S)}$ and centered at any point $X(t) \in \mathbf{R}^{(N \times S)}$ :

$$
\widehat{f}_{P}\left(x \mid i_{1}, \ldots, i_{S} ; r\right)=\frac{1}{P} \int_{0}^{P} \frac{1}{h^{(N \times S)}} \mathbf{I}_{A}\left(\frac{x-X(t)}{h}\right) d t .
$$

In general, the (kernel) density estimator of $f\left(X(t) \mid i_{1}, \ldots, i_{S} ; r\right)$ is defined by introducing a positive $(N \times S)$-dimensional kernel function $K$ with the integral $\int_{\mathbf{R}^{(N \times S)}} K(x) d x=1$ :

$$
\widehat{f}_{P}\left(x \mid i_{1}, \ldots, i_{S} ; r\right)=\frac{1}{P h^{(N \times S)}} \int_{0}^{P} K\left(\frac{x-X(t)}{h}\right) d t .
$$

The multidimensional uniform distribution is used to produce the rectangular estimator in (5). To get a smooth density function, a smooth kernel such as multi-dimensional Gaussian should be in (6).

\section{Example: application of our method on synthetic data}

To illustrate this approach, we applied it on synthetic data. Below we describe this example.

Sensors used in falls detection typically provide information about acceleration and balance. Such sensors produce signals that are essentially combinations of sinusoids, which are periodic functions. Because the sensor signals are sampled, the sampled signals become discrete-time and non-smooth. For simplicity, we consider signals that are periodic, piece-wise linear, and discrete-time as representative synthetic data in this example. Moreover, we assume two sensors each recording a 1-dimensional signal, i.e., $S=2$ and $N_{1}=N_{2}=N=1$.

Suppose that sensor 1 signal is the following function $a_{1}(\cdot)$ with period 8 :

$$
a_{1}(t \pm 8 k)=\left\{\begin{aligned}
t, t & =0,1,2 \\
4-t, t & =2,3,4,5,6 \\
t-8, t & =6,7,8
\end{aligned}\right.
$$


where $k=0,1,2, \ldots$.

Similarly, suppose that sensor 2 signal is given by the function $a_{2}(\cdot)$ with period 8 that is shown below:

$$
a_{2}(t \pm 8 k)=\left\{\begin{array}{l}
t-1, t=0,1,2,3 \\
5-t, t=3,4,5,6,7 \\
t-9, t=7,8
\end{array}\right.
$$

where $k=0,1,2, \ldots$

Many practical methods for falls detection from wearable sensors are threshold-based. The threshold-based procedures are able to detect falls with high probability by identifying high amplitude signals. Likewise, we assume here that the outcome of a loss of balance (i.e., a fall or a regained balance) depends on the amplitude of the signal. But, for simplicity, we think of such dependence as deterministic rather than probabilistic. Specifically, we suppose that a fall or a no-fall (i.e., a complete regaining of balance) happens based on the amplitude of either signal at trigger time $T$, i.e.,

$$
\begin{aligned}
\{\text { Fall at } H\} & \Leftrightarrow\left\{\left|a_{1}(T)\right| \geq \theta_{1}\right\} \cup\left\{\left|a_{2}(T)\right| \geq \theta_{2}\right\} \\
\{\text { No-Fall at } H\} & \Leftrightarrow\left\{\left|a_{1}(T)\right|<\theta_{1}\right\} \cap\left\{\left|a_{2}(T)\right|<\theta_{2}\right\},
\end{aligned}
$$

where $\theta_{i}, i=1,2$ is the falling threshold for sensor $i$. For instance, if $\theta_{i}=2$, $i=1,2$ then we have:

(i) No-falls at $T=0 \pm 8 k, 1 \pm 8 k, 4 \pm 8 k, 5 \pm 8 k$ (with $k=0,1,2, \ldots$ );

(ii) Falls detected by sensor 1 at $T=2 \pm 8 k, 6 \pm 8 k$ (with $k=0,1,2, \ldots$ );

(iii) Falls detected by sensor 2 at $T=3 \pm 8 k, 7 \pm 8 k$ (with $k=0,1,2, \ldots$ ).

Figure 1 shows signals $a_{1}(t)$ and $a_{2}(t)$ in $(7)$ and (8), respectively, plotted at times $t=-8,-7, \ldots, 8,9,10$. We assume that either signal can detect a loss of balance. Furthermore, the fall is predicted when the condition (9) is satisfied during the prediction time period $[t, t+\tau]$, i.e., $\exists T \in[t, t+\tau]:\left\{\left|a_{1}(T)\right| \geq\right.$ $\left.\theta_{1}\right\} \cup\left\{\left|a_{2}(T)\right| \geq \theta_{2}\right\}$.

As shown in Figure 1, we will use the observations $X(t)=\left(a_{1}(t), a_{2}(t)\right)$ at times $t=-8,-7, \ldots,-2,-1$ as the training dataset and $\{X(t), t=0,1, \ldots, 7\}$ as the testing data. In this example, let us consider the prediction interval with $\tau=1$. Then Table 1 presents the observations $X(t)$, the prediction intervals $I=[t, t+\tau]$, and the known rankings of fall $(\mathrm{F})$ and no-fall $(\mathrm{N})$ outcomes in rows $2,3,4,5$, and 6 , respectively. The rankings of fall and no-fall outcomes are assigned in decreasing order of likelihood, i.e., rank 1 represents a very likely outcome, whereas 2 means that the outcome is not likely (or at least less likely than the other outcome). Because $S=2$ we have two values $r_{1} r_{2}$ (with $r_{1}, r_{2} \in\{1,2\}$ ) as the ranking of each outcome. For instance, the ranking of the fall outcome (F) at time $t=-7$ is 12 . This means that one expects $\mathrm{F}$ to be more likely than $\mathrm{N}$ based on the information from sensor 1, but less likely than $\mathrm{N}$ according to the sensor 2 signal. 


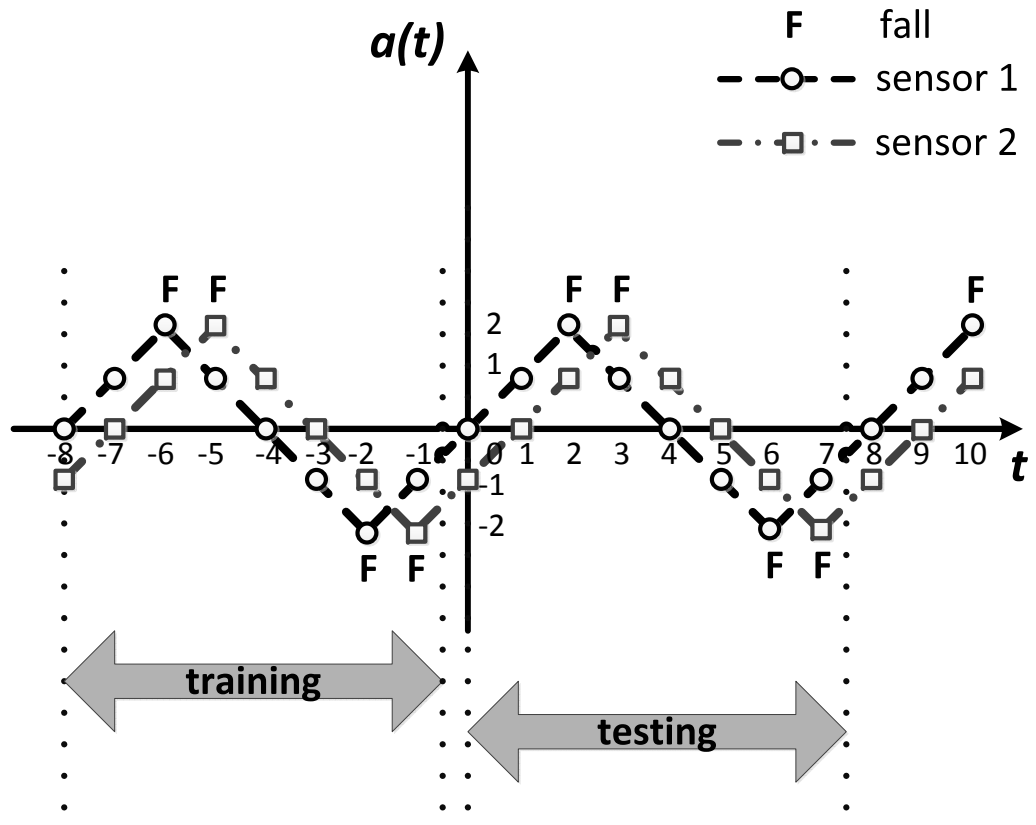

Fig. 1 Sensor 1 signal $a_{1}(t)$ and sensor 2 signal $a_{2}(t)$ are shown. Training dataset contains observations at $t=-8,-7, \ldots,-2,-1$. Testing dataset includes observations at $t=0,1, \ldots, 6,7$. Fall events $\mathrm{F}$ happen at times $t=-6,-5,-2,-1,2,3,6,7,10$

Table 1 The prediction intervals $I=[t, t+\tau]$ and the observed rankings of fall $(\mathrm{F})$ and no-fall $(\mathrm{N})$ for the testing dataset are used in estimation of density functions

\begin{tabular}{c|rrrrrrrr}
\hline$t$ & -8 & -7 & -6 & -5 & -4 & -3 & -2 & -1 \\
\hline$X(t)$ & $(0,-1)$ & $(1,0)$ & $(2,1)$ & $(1,2)$ & $(0,1)$ & $(-1,0)$ & $(-2,-1)$ & $(-1,0)$ \\
$I$ & {$[-8,-7]$} & {$[-7,-6]$} & {$[-6,-5]$} & {$[-5,-4]$} & {$[-4,-3]$} & {$[-3,-2]$} & {$[-2,-1]$} & {$[-1,0]$} \\
$\mathrm{F}$ & 22 & 12 & 11 & 21 & 22 & 12 & 11 & 21 \\
$\mathrm{~N}$ & 11 & 21 & 22 & 12 & 11 & 21 & 22 & 12 \\
\hline
\end{tabular}

From Table 1, we compute the estimated density functions using the histogram estimator (4). Specifically, we obtain:

$$
\begin{aligned}
& \widehat{f}(x \mid F 22)=\frac{1}{8} I_{\{(0,-1),(0,1)\}}(x)=\widehat{f}(x \mid N 11) \\
& \widehat{f}(x \mid F 12)=\frac{1}{8} I_{\{(1,0),(-1,0)\}}(x)=\widehat{f}(x \mid N 21) \\
& \widehat{f}(x \mid F 11)=\frac{1}{8} I_{\{(2,1),(-2,-1)\}}(x)=\widehat{f}(x \mid N 22) \\
& \widehat{f}(x \mid F 21)=\frac{1}{8} I_{\{(1,2),(-1,-2)\}}(x)=\widehat{f}(x \mid N 12)
\end{aligned}
$$

Plugging the values of estimated density functions on the testing data into (3), we get the values of coefficients $L_{r i_{1} \ldots i_{S}}$ in (2) for each point of the testing sample. In this example, there are two types of risk exposures and two sensors, so $R=2$ and $S=2$. Recall that the dataset used for testing consists 
Table 2 The predicted rankings of fall $(\mathrm{F})$ and no-fall $(\mathrm{N})$ for the training dataset are obtained by solving the optimization problem

\begin{tabular}{c|rrrrrrrr}
\hline$t$ & 0 & 1 & 2 & 3 & 4 & 5 & 6 & 7 \\
\hline$X(t)$ & $(0,-1)$ & $(1,0)$ & $(2,1)$ & $(1,2)$ & $(0,1)$ & $(-1,0)$ & $(-2,-1)$ & $(-1,0)$ \\
$\mathrm{F}$ & 22 & 12 & 11 & 21 & 22 & 12 & 11 & 21 \\
$\mathrm{~N}$ & 11 & 21 & 22 & 12 & 11 & 21 & 22 & 12 \\
\hline
\end{tabular}

of the $X(t)$ values at $t=0,1, \ldots, 7$. As a result, for each time $t=0,1, \ldots, 6,7$, we obtain the respective multidimensional assignment problem:

$$
\max _{\pi_{1}, \pi_{2} \in \Pi_{2}} L_{F \pi_{1}(F) \pi_{2}(F)}+L_{N \pi_{1}(N) \pi_{2}(N)},
$$

where $\Pi_{2}=\left\{\left(\begin{array}{l}1 \\ 2\end{array}\right),\left(\begin{array}{l}2 \\ 1\end{array}\right)\right\}$.

For instance, when $t=1$, the measurements from the sensors give us the data point $X(t)=(1,0)$. Plugging $x=(1,0)$ into (11), (13), and (14), we have

$$
\widehat{f}(x \mid F 22)=\widehat{f}(x \mid N 11)=\widehat{f}(x \mid F 11)=\widehat{f}(x \mid N 22)=\widehat{f}(x \mid F 21)=\widehat{f}(x \mid N 12)=0,
$$

leading to

$$
L_{F 22}=L_{F 11}=L_{F 21}=L_{N 11}=L_{N 22}=L_{N 12}=\ln 0=-\infty .
$$

Likewise, plugging $x=(1,0)$ into $(12)$, we get

$$
\widehat{f}(x \mid F 12)=\widehat{f}(x \mid N 21)=\frac{1}{8},
$$

and so

$$
L_{F 12}=L_{N 21}=\ln \frac{1}{8}=-\ln 8 .
$$

Using these coefficients in the maximization problem (15) and $-\ln 8>-\infty$, it is obvious that the solution of the MAP for $t=1$ is

$$
\begin{array}{lll}
F & 1 & 2 \\
N & 2 & 1
\end{array}
$$

or

$$
\Pi=\left(\begin{array}{ll}
1 & 2 \\
2 & 1
\end{array}\right)
$$

where $r=1$ is $\mathrm{F}$ and $r=2$ is $\mathrm{N}$.

Maximizing the likelihood of the observed data $X(t)$ for each remaining point $t$ of the training data in the same way we did for $t=1$ with $X(t)=(1,0)$, we obtain the predicted rankings shown in Table 2 . 


\section{Discussion}

In this section we discuss the described approach and the presented example, the limitations of this study, and the future research directions.

First, it is important to note that the developed approach is a general method for sensor-based surveillance of exposure to falls or near falls. It works by combining the data from multiple (i.e., two or more) wearable sensors to establish a ranking of distinct types of exposures. Types of exposures can be defined broadly by the approach user depending on the goals of study. For instance, when focus is on understanding falls and near falls during walking, one may want to differentiate among slips, trips, usual walking, and obstacle avoidance. Having the sensors positioned in different location on the body could provide complimentary information on whether some type or types of exposures are more likely, similarly to the falls classification gains observed in [11]. For instance, high acceleration measurements from an acceleromter sensor can have different interpretations based on the specific location of this sensor on a human body. Furthermore, different exposures can result in similar measurements for a single sensor. In other words, multiple interpretations are possible due to uncertainty about which exposure supplied the sensor data. By combining information from several locations (e.g., ankles, hips, wrists), it may be possible to reduce the uncertainty about the exposures given the measurements.

Second, we remark that the presented example is a simplification. It is intended to illustrate how the instance of optimization problem is derived for a simplified case. It should not be interpreted as the indication that (i) the approach is developed for falls detection, or (ii) threshold algorithms should be used for training in the application of our approach. In fact, our approach is developed for a purpose of situational awareness rather than more traditional falls detection. The application of the approach on the current sensor data results in the ranking of exposures. The current ranking of distinct types of exposures to possible falls provides awareness about which types of situations are most likely at the moment.

Thresholding $[12,13]$ was used in the example to get the assignment of the ranking to the data. This assignment was later used as the training data set, from which the coefficients' values of the problem instance were computed. In real-world application, this would not constitute a suitable approach for obtaining the training data set. Instead, the training data should be assigned the respective ranking of exposures based on either the rankings assigned by trained qualified raters, or by the rankings based on the analyses of dynamic and kinematic properties of observed biomechanics, or a combination of both.

The focus of the current study is on establishing a formal method for ranking the distinct exposures to falls and near falls. The current study has several important limitations. First of all, the validity of the presented optimizationbased approach needs to be assessed on real-world data. Second, the approach does not address the non-stationary noise in the signal that is present in wearable sensors' measurements. The practical usefulness of this approach may 
be improved by suitable preprocessing of signals to eliminate the noise artifacts [28]. The preprocessing techniques may include spectral analysis methods (such as fast Fourier transforms), statistics-based metrics (e..g., crosscorrelation), and string matching techniques. Furthermore, various measures [29] of the dynamic stability of human locomotion could be computed from the raw signals and used as the signals in the proposed approach.

Third, the optimization approach is developed for training on an individual user of the wearable sensor system and assumes the sufficient training data. In its current form, it is not applicable to multiple subjects or situations when the subject's movement biomechanics change significantly (e.g., as a result of a stroke). The new approach does not specifically address the inherent variability in human movement among subjects or due to a recently acquired disability. Additional research is needed to understand how to address human movement variability and provide guidelines on the amount of data needed for proper training of the model for different numbers of sensors and exposures.

\section{Conclusions}

We studied a problem of situational risk monitoring and situational awareness of falls and near falls with multiple complementary sensors. Specifically, we proposed a formal method for ranking distinct types of exposures to falls or near falls. Whereas sensor-based falls detection has received a lot of attention, there is a clear need for the development of approaches for situational awareness about environmental hazards and circumstances of falls and near falls events. We proposed a novel optimization-based approach for falls risk monitoring and situational assessment, which incorporates various estimation methods from classical statistics. Our approach is developed specifically for fusion of information about specific fall risks from multiple sensors. In contrast to neural networks and other difficult-to-interpret data mining techniques (often used in fall detection with sensors), our approach naturally enables situational awareness about circumstances surrounding falls and near falls.

Acknowledgements The author gratefully acknowledges the support from the National Science Foundation grant EEC-1342415.

\section{References}

1. Global health and ageing, WHO, US National Institute of Aging, October 2011, http://www.who.int/ageing/publications/global_health.pdf, Apr. 11, 2013.

2. J.A. Stevens, P.S. Corso, E.A. Finkelstein, T.R. Miller, The costs of fatal and non-fatal falls among older adults, Injury Prevention 12, no. 5 (2006) 290-295.

3. Institute of Medicine (US) Division of Health Promotion and Disease Prevention; Berg R.L., Cassells J.S., editors. The Second Fifty Years: Promoting Health and Preventing Disability. Washington (DC): National Academies Press (US); 1992. 15, Falls in Older Persons: Risk Factors and Prevention. Available from: http://www.ncbi.nlm.nih.gov/books/NBK235613/ 
4. M.E. Tinetti, M. Speechley, and S.F. Ginter. Risk factors for falls among elderly persons living in the community. New England journal of medicine 319, no. 26 (1988): 17011707.

5. I. Maidan, T. Freedman, R. Tzemah, N. Giladi, A. Mirelman, J. Hausdorff. Introducing a new definition of a near fall: Intra-rater and inter-rater reliability. Gait 85 posture 39, no. 1 (2014):10.1016/j.gaitpost.2013.07.123. doi:10.1016/j.gaitpost.2013.07.123.

6. J.M. Srygley, T. Herman, N. Giladi, J.M. Hausdorff. Self-report of missteps in older adults: a valid proxy of fall risk? Arch Phys Med Rehabil. 90, no. 5 (2009): 786-92.

7. N. Noury, A. Fleury, P. Rumeau, A.K. Bourke, G.O. Laighin, V. Rialle, J.E. Lundy, Fall detection-principles and methods, in: Engineering in Medicine and Biology Society, 2007. EMBS 2007, 29th Annual International Conference of the IEEE, IEEE, 2007, pp. $1663-1666$.

8. T. Shany, S.J. Redmond, M.R. Narayanan, N.H. Lovell, Sensors-based wearable systems for monitoring of human movement and falls, Sensors Journal, IEEE 12, no. 3 (2012) $658-670$

9. Q. Li, J.A. Stankovic, M.A. Hanson, A.T. Barth, J. Lach, G. Zhou, Accurate, fast fall detection using gyroscopes and accelerometer-derived posture information, in: Sixth International Workshop on Wearable and Implantable Body Sensor Networks, 2009. BSN 2009, IEEE, 2009, pp. 138-143.

10. D. Giansanti, V. Macellari, G. Maccioni, New neural network classifier of fall-risk based on the Mahalanobis distance and kinematic parameters assessed by a wearable device, Physiol. Meas. 29 (2008) N11-N19.

11. O. Aziz, S.N. Robinovitch, An analysis of the accuracy of wearable sensors for classifying the causes of falls in humans, IEEE Transactions on Neural Systems and Rehabilitation Engineering 19, no. 6 (2011) 670-676.

12. A.K. Bourke, J.V. OBrien, and G.M. Lyons. Evaluation of a threshold-based tri-axial accelerometer fall detection algorithm. Gait \& Posture 26, no. 2 (2007): 194-199.

13. A.K. Bourke, and G.M. Lyons. A threshold-based fall-detection algorithm using a biaxial gyroscope sensor. Medical Engineering $\&$ Physics 30, no. 1 (2008): 84-90.

14. T. Zhang, J. Wang, L. Xu, and P. Liu. Fall detection by wearable sensor and one-class SVM algorithm. In Intelligent Computing in Signal Processing and Pattern Recognition, pp. 858-863. Springer Berlin Heidelberg, 2006.

15. F. Bagalà, C. Becker, A. Cappello, L. Chiari, K. Aminian, J.M. Hausdorff, W. Zijlstra, and J. Klenk. Evaluation of accelerometer-based fall detection algorithms on real-world falls. PloS one 7, no. 5 (2012): e37062.

16. C.C. Yang, and Y.-L. Hsu. A review of accelerometry-based wearable motion detectors for physical activity monitoring. Sensors 10, no. 8 (2010): 7772-7788.

17. M. Marschollek, A. Rehwald, K.H. Wolf, M. Gietzelt, G. Nemitz, H.M. Zu Schwabedissen, and R. Haux. Sensor-based fall risk assessmentan expert 'to go'. Methods Inf Med 50, no. 5 (2011): 420-426.

18. J. Howcroft, J. Kofman, and E.D. Lemaire. Review of fall risk assessment in geriatric populations using inertial sensors. J Neuroeng Rehabil 10, no. 1 (2013): 10-91.

19. W. Zijlstra, and K. Aminian. Mobility assessment in older people: new possibilities and challenges. European Journal of Ageing 4, no. 1 (2007): 3-12.

20. W. Zijlstra, R.W. Bisseling, S. Schlumbohm, and H. Baldus. A body-fixed-sensor-based analysis of power during sit-to-stand movements. Gait \& Posture 31, no. 2 (2010): $272-278$.

21. A. Weiss, I. Shimkin, N. Giladi, and J.M. Hausdorff. Automated detection of near falls: algorithm development and preliminary results. BMC Research Notes 3, no. 1 (2010): 62 .

22. W.P. Pierskalla. The multidimensional assignment problem, Operations Research 16 (1968) 422-431.

23. A.R. Kammerdiner, Multidimensional assignment problem, in: Encyclopedia of Optimization, Springer, 2009, pp. 2396-2402.

24. G. Casella, R.L. Berger, Statistical inference, second ed., Pacific Grove, CA: Duxberry, 2002.

25. J.W. Harris, H. Stocker, Maximum likelihood method, in: Handbook of Mathematics and Computational Science, Springer-Verlag, New York, 1998, p. 824. 
26. NIST/SEMATECH e-Handbook of Statistical Methods, http://www.itl.nist.gov/div898/handbook/, Nov. 15, 2014

27. N. Thaper, S. Guha, P. Indyk, N. Koudas, Dynamic multidimensional histograms, in: Proceedings of the 2002 ACM SIGMOD international conference on Management of data, ACM, June 2002, pp. 428-439.

28. D. Figo, P.C. Diniz, D.R. Ferreira, and J.M.P. Cardoso. Preprocessing techniques for context recognition from accelerometer data. Personal and Ubiquitous Computing 14 no. 7 (2010): $645-662$.

29. S.M. Bruijn, O.G. Meijer, P.J. Beek, and J.H. Van Dien. Assessing the stability of human locomotion: a review of current measures. Journal of The Royal Society Interface 10 no. 83 (2013): 20120999 